\title{
Moderately Hypofractionated Intensity Modulated Radiation Therapy with Simultaneous Integrated Boost for Prostate Cancer: Five-Year Toxicity Results from a Prospective Phase I/II Trial
}

\section{Anthony Ricco}

Virginia Commonwealth University Medical Center https://orcid.org/0000-0003-3916-2747

Nitai Mukhopadhyay

Virginia Commonwealth University Health, Department of Biostatistics

Xiaoyan Deng

Virginia Commonwealth University Health, Department of Biostatistics

Diane Holdford

Virginia Commonwealth University Medical College Official Bookstore

\section{Vicki Skinner}

Hunter Holmes McGuire VA Medical Center

\section{Siddharth Saraiya}

Virginia Commonwealth University Health, Department of Radiation Oncology

Drew Moghanaki

Virginia Commonwealth University, Department of Radiation Oncology

\section{Mitchell Anscher}

Virginia Commonwealth University Health, Department of Radiation Oncology

Michael Chang ( $\nabla$ michael.chang@vcuhealth.org )

Virginia Commonwealth University Health, Department of Radiation Oncology

\section{Research}

Keywords:

Posted Date: May 19th, 2020

DOl: https://doi.org/10.21203/rs.3.rs-28814/v1

License: (1) This work is licensed under a Creative Commons Attribution 4.0 International License. Read Full License 
Version of Record: A version of this preprint was published at Frontiers in Oncology on August 21st, 2020. See the published version at https://doi.org/10.3389/fonc.2020.01686. 


\section{Abstract}

Background In this phase I/II trial, five-year physician-assessed toxicity and patient reported quality of life data is reported for patients undergoing moderately hypofractionated intensity modulated radiation therapy (IMRT) for prostate cancer using a simultaneous integrated boost (SIB) and pelvic lymph node (LN) coverage. Materials and Methods Patients with T1-T2 localized prostate cancer were prospectively enrolled, receiving risk group based coverage of prostate +/- seminal vesicles (SVs) +/- pelvic lymph nodes (LNs). Low risk (LR) received $69.6 \mathrm{~Gy} / 29$ fractions to the prostate, while intermediate risk (IR) and high risk (HR) patients received $72 \mathrm{~Gy} / 30 \mathrm{fx}$ to the prostate and $54 \mathrm{~Gy} / 30 \mathrm{fx}$ to the SVs. If predicted risk of $\mathrm{LN}$ involvement $>15 \%, 50.4 \mathrm{~Gy} / 30 \mathrm{f} x$ was delivered to pelvic $\mathrm{LNs}$. Androgen deprivation therapy was given to IR and HR patients. Results There were 55 patients enrolled and 49 patients evaluable at a median follow up of 60 months. Included were 11 (20\%) LR, 23 (41.8\%) IR, and 21 (38.2\%) HR patients. Pelvic LN treatment was given in 25 patients (51\%). Prevalence rates of late grade $2 \mathrm{Gl}$ toxicity at 1,3 , and 5 years was $5.8 \%, 3.9 \%$, and $5.8 \%$ respectively, with no grade 3 events. Prevalence rates of late grade $2 \mathrm{GU}$ toxicity at 1,3 , and 5 years rates were $15.4 \%, 7.7 \%$, and $13.5 \%$ respectively, with three grade 3 events $(5.8 \%)$. The biochemical relapse free survival at 5 years was $88.3 \%$. There were no local, regional, or distant failures, with all patients still alive at last follow up. Conclusions Moderate hypofractionation of localized prostate cancer utilizing a SIB technique and LN coverage produces tolerable acute/late toxicity. Given equivalent efficacy between moderate hypofractionation schedules, the optimal regimen will be determined by longterm toxicity reported from both the physician and patient perspective.

\section{Introduction}

External beam radiation treatment (EBRT) has been a standard treatment in organ confined prostate cancer with high rates of biochemical control and acceptable rates of acute and late toxicity with up to 20-year follow up. ${ }^{1}$ Advances in the precision and conformality of external beam radiation delivery have allowed dose escalation to decrease biochemical failure rates (BF) while reducing gastrointestinal (GI) and genitourinary $(\mathrm{GU})$ toxicities, in addition to possibly decreasing prostate cancer specific mortality (PCSM). ${ }^{1,2}$ These advances occurred during the same time period that radiobiologic models suggested prostate cancer's would be more sensitive to hypofractionation than conventional fractionation which in turn led to the successful completion of multiple randomized controlled trials comparing standard to moderate hypofractionation. These trials demonstrated equivalent BF, PCSM, overall survival (OS). ${ }^{3-13}$ In 2018, this led to a joint ASTRO, ASCO, and AUA guideline recommending moderate hypofractionation be offered across low, intermediate, and high risk groups regardless of age, comorbidity, and urinary function. ${ }^{14}$

Consensus opinion is that moderate hypofractionation has a similar risk of acute $\mathrm{GU}$ and late $\mathrm{GU} / \mathrm{GI}$ toxicity compared to conventional EBRT with higher rates of acute GI toxicity. ${ }^{14}$ However, the optimal hypofractionation scheme remains unknown, as there was significant heterogeneity between moderate hypofractionation schedules without one demonstrating superiority. In addition, few trials had included 
pelvic nodal treatment using a simultaneous integrated boost (SIB) technique. Our institutional policy in 2010 was to deliver between 75.6 and $77.4 \mathrm{~Gy}$ in $1.8 \mathrm{~Gy}$ fractions to the prostate. We opened a phase I/II trial at that time to deliver 69.6 to $72 \mathrm{~Gy}$ at $2.4 \mathrm{~Gy}$ per fraction in 29 to 30 fractions to the prostate, while elective seminal vesicle (SV) at $1.8 \mathrm{~Gy} / \mathrm{fx}$ and pelvic nodal coverage at $1.68 \mathrm{G} / \mathrm{fx}$ were delivered depending on risk of involvement using an SIB technique.

With similar efficacy between moderate hypofractionation trials, prospectively collected long-term toxicity reported from both the physician and patient perspective will help to distinguish potential optimal regimens. In this manuscript, we provide mature 5 year toxicity data in addition to patient reported outcomes for a moderately hypofractionated schedule utilizing pelvic nodal treatment with simultaneous integrated boost (SIB) technique.

\section{Methods}

\section{STUDY DESIGN}

This phase I/II single-institutional trial sought to assess the rates of acute and late toxicity with a secondary endpoint of biochemical control with risk-adapted moderately hypofractionated IMRT (ClinicalTrials.gov identifier: XXXX). Eligible patients had clinically node negative adenocarcinomas of the prostate, stage T1-T2, with Karnofsky Performance Status (KPS) >= 80. Prior to enrollment, patients underwent pretreatment evaluation with history and physical, digital rectal examination, complete blood count, and liver function tests. Serum prostate-specific antigen (PSA) and prostatic biopsies were required within 12 months of enrollment. Patients also had pre-study, post-study, and weekly during treatment assessments of $\mathrm{Gl}, \mathrm{GU}$, and erectile function which were both physician and patient assessed. Patients were stratified into low risk (LR), intermediate risk (IR), and high risk (HR) groups according to National Comprehensive Cancer Network (NCCN, version 1.2010) guidelines, with high risk patients receiving a computed tomography (CT) scan of the abdomen and pelvis and Tc99m-MDP bone scans to rule out metastatic disease.

LR patients received 69.6 Gy to the prostate alone in 29 fractions of 2.4 Gy each. The IR and HR patients received $72 \mathrm{~Gy}$ to the prostate in 30 fractions of $2.4 \mathrm{~Gy}$ each and $54 \mathrm{~Gy}$ in 30 fractions of $1.8 \mathrm{~Gy}$ fractions to the proximal $1 \mathrm{~cm}$ seminal vesicle (SV) using a simultaneously integrated boost (SIB) technique. Patients received pelvic nodal irradiation to $50.4 \mathrm{~Gy}$ in 30 fractions of $1.68 \mathrm{~Gy}$ with SIB technique if their calculated Roach formula risk of LN involvement $>=15 \% .{ }^{15}$ Androgen deprivation therapy (ADT) was given two months prior to the initiation of radiotherapy for a total duration of 6 months to IR patients and 36 months to HR patients.

Intraprostatic fiducial markers were placed transperineally in all patients for daily image guidance. CT simulation images were captured from the L1 vertebral body superiorly to mid-thigh inferiorly with 3-mm slice thickness. Patients were instructed to maintain a full bladder and empty rectum for simulation and daily treatments. The prostate was defined as the GTV and CTV; the proximal $1 \mathrm{~cm}$ of SV was contoured 
separately. CTV to PTV expansion was $7 \mathrm{~mm}$ in all directions except for $3 \mathrm{~mm}$ posteriorly. Pelvic nodal PTV was defined as a $10 \mathrm{~mm}$ expansion in all directions off external, internal, and common iliac vessel contours.

Organs at risk (OAR) included rectum, posterior rectum, bladder, small bowel, femoral heads, and skin contoured according to national guidelines, with dose constraints listed in Table $1 .{ }^{16}$ Rectum was contoured from the anterior flexion of the recto-sigmoid superiorly to the level of the ischial tuberosity inferiorly. Posterior rectum consisted of the posterior half of the rectum on each axial CT slice separating the midway point between the anterior-most and posterior-most aspects of the rectal contour on each axial CT slice. Daily image guidance with kilovoltage or megavoltage orthogonal imaging, Calypso beacons (Varian, Palo Alto, CA), or cone beam CT was performed on all patients before treatment.

\section{TOXICITY AND ANALYSIS}

The primary endpoint of this phase I/II study was to measure the physician-reported cumulative rate of late grade 2 or higher GI and GU toxicity. Secondary endpoints included acute GI/GU toxicity, transient late GI/GU toxicity, prevalence of late GI/GU toxicity at last follow up, patient reported toxicity/quality of life indicators, and biochemical relapse free survival (bRFS). Acute toxicity was defined as onset and resolution within 90 days of treatment. Late toxicity was defined as unresolved acute toxicity or onset beyond 90 days, with patients requiring medication at or beyond 90 days defined as having late grade 2 GU toxicity. A historic rate of grade 2 toxicity of $14.4 \%$ was utilized for comparison, with a rate of $30 \%$ or

higher deemed as unacceptable toxicity per protocol. The Data and Safety Monitoring Committee would stop the protocol if the rate of late grade 3 or higher GI or GU toxicity was greater than $7 \%$. The study aimed to accrue 55 patients for an $86 \%$ power utilizing a 1-sample, 1-sided Fisher exact test at the $5 \%$ level of significance allowing for a $10 \%$ dropout rate.

The physician-reported Common Terminology Criteria for Adverse Events (CTCAE, version 3.0), the patient-reported International Prostate Symptom Score (IPSS), and the patient-reported International Index of Erectile Function 5 (IIEF) represented the data collection mechanisms of monitoring acute and late GI and GU toxicity. Toxicity was assessed prior to EBRT as baseline assessments, weekly during EBRT, and at every follow up visit. Follow up schedule included initial visit 4-6 weeks after treatment, every 4 months for 3 years, and then semi-annually for 2 years. All analyses were performed in statistical software R v3.2.1 (R Foundation for Statistical Computing, Vienna, Austria).

\section{Results}

Between June 2010 and June 2013, 55 patients were enrolled and data from 52 patients were available for the analyses. Two patients were enrolled and then withdrew consent before receiving any radiotherapy. The final patient developed grade 3 diarrhea felt to be infectious in nature midway through treatment, and discontinued therapy at that time. Three of the 52 patients who completed radiotherapy did not complete the minimum of 12 months of follow up which left 49 patients evaluable for 
biochemical recurrence and toxicity. The median follow up of this group was 60 months (range 8-60 months), as all patients were followed only to a maximum of 60 months.

Patient demographics and characteristics can be found in Table 1. Eleven patients had LR disease, 18 had IR disease with nodal risk $<15 \%, 5$ had IR disease with nodal risk $=>15 \%$, and 21 had HR disease. Twenty-five patients received prostate and pelvic nodal treatment with SIB technique, with 24 receiving prostate-only treatment. Ultimately, 44 out of $55(80 \%)$ patients enrolled received ADT.

\section{Physician-reported toxicity}

Late GI toxicity consisted mostly of self-limited grade $1 / 2$ toxicities which resolved over time. At 5 years, the cumulative incidence of late grade 2 or higher GI toxicity was $22.6 \%$ (Fig. 1). The percentage of patients experiencing a late grade $2 \mathrm{GI}$ toxicity at 1,3 , and 5 years was $5.8 \%, 3.8 \%$, and $5.8 \%$ respectively (Fig. 2). Of the 12 patients who were found to have late GI toxicities of grade 2 or higher, 1 reported anal incontinence, 5 reported flatulence, 4 reported diarrhea, and 2 reported either rectal bleeding or proctitis. There were no patients who with unresolved late grade $3 \mathrm{GI}$ toxicity at last follow up. Late grade 2 or higher $\mathrm{Gl}$ toxicities that did not resolve by 60 months included 3 out of 52 patients (5.8\%); 1 reported flatulence, 1 reported anal incontinence, and 1 reported diarrhea. All three of these toxicities did resolve with subsequent follow up at the 74,61 , and 67 month timepoint after treatment respectively.

At 5 years, the cumulative incidence of late grade 2 or higher GU toxicity was $38.2 \%$ (Fig. 1). The percentage of patients experiencing a late grade $1 \mathrm{GU}$ toxicity at 1,3 , and 5 years was $25 \%, 30.1 \%$, and $5.8 \%$ respectively, with grade 2 rates at $15.4 \%, 7.7 \%$, and $13.5 \%$ respectively (Fig. 2 ). There were 3 patients (5.8\%) who experienced grade $3 \mathrm{GU}$ toxicity, which included bladder spasms, urinary incontinence, and cystitis. There were $4(7.7 \%)$ late grade 2 or higher toxicities which did not resolve by 60 months; 1 with radiation cystitis, 1 with urethral stricture, 1 with urinary retention, and 1 unknown which all resolved at the $74,61,68$, and 61 month timepoint after treatment respectively.

In the subset of patients who received pelvic nodal radiation therapy, there was a trend towards lower rates of late GU and GI toxicity, which can be seen in Fig. 2. For example, the prevalence of any late GI toxicity at 1,3 , and 5 years was $42.3 \%$ vs. $30.8 \%, 26.9 \%$ vs. $26.9 \%$, and $15.4 \%$ vs. $11.5 \%$ for non-nodal treatment vs. nodal treatment respectively. In addition, the prevalence of any late GU toxicity at 1,3 , and 5 years was $51.8 \%$ vs. $30.8 \%, 42.3 \%$ vs. $34.6 \%$, and $30.7 \%$ vs. $7.7 \%$ for non-nodal treatment vs. nodal treatment respectively. There were no statistically significant differences in cumulative rates of late $\mathrm{GI}$ or GU toxicity between patients who did and did not receive pelvic nodal radiation therapy with SIB technique.

Patient Reported Toxicity / Quality of Life Indicators

Baseline patient-reported IPSS score was on average 11.3 prior to radiotherapy. During treatment this rose to a mean of 15.0, and subsequently fell back to pretreatment baseline at 1 year with an average score of 11.9. The 3 and 5 year IPSS scores remained at baseline, 10.8 and 11.7 respectively on average (Fig. 3 ). 
Baseline patient-reported IIEF score was on average 28.8 prior to radiotherapy. During treatment this fell to a mean 18.6 at 4 months post treatment coinciding with ADT use. Scores increased gradually over time to near baseline levels (24.8) by 5 years (Fig. 4).

Tumor Control

The bRFS at 5 years for the cohort was $88.3 \%$ (Fig. 5). There were no local, regional, or distant failures, with all patients still alive at last follow up.

\section{Discussion}

Moderate hypofractionation in prostate cancer has now been accepted and recommended to be offered across all risk groups. ${ }^{14}$ There remains uncertainty however about the optimal dose-fractionation, as significant heterogeneity among moderate hypofractionation trials exist. In addition, many trials' toxicity outcomes are difficult to interpret. ${ }^{3}$ For example, both the PROFIT and CHHiP trials show no difference in late GI or GU toxicity between conventional and moderate hypofractionation arms, however the RTOG 0415 trial showed a significant difference and the MD Anderson trial showed trend towards worse late $\mathrm{GI}$ toxicity in the hypofractionated arms. $4,5,10,12$ This current prospective clinical trial adds to the data on toxicity rates with moderate hypofractionation, strengthened by the inclusion of hypofractionated pelvic nodal treatments utilizing modern radiotherapy techniques with IMRT using an SIB technique and daily image guidance which has only recently been introduced into routine clinical care. As moderate hypofractionation's efficacy in localized prostate cancer treatment has now been accepted, prospectively collected long-term toxicity and patient quality of life data will help to distinguish the optimal dose fractionation schedule.

With a median follow up of 5 years, the updated toxicity data from this study once again demonstrates physician and patient-reported late $\mathrm{GI}$ and GU toxicity rates that are comparable to similar series. The vast majority of patients experienced transient grade 2 or higher late $\mathrm{GI}$ and GU toxicities which resolved over time. While the physician-reported GU toxicity exceeded the upper limit of tolerance by trial design, there were only $7.7 \%$ late grade 2 or higher toxicities (4 patients) and $5.8 \%$ of late grade 2 or higher toxicities ( 3 patients) which did not resolve by 5 years. The grade $3 \mathrm{GU}$ toxicity rate was $5.8 \%$, comparable to other hypofractionation trials such as $3.5 \%$ on RTOG 0415 and $0.7 \%$ in the PROFIT trial . ${ }^{4,10}$ Neither the GU nor GI toxicity rates increased significantly between 2-5 years post-treatment, lending evidence for long term safety. However, it is clear that even at 5 years of follow up, there are grade 2 toxicities $(5.8 \% \mathrm{GI}$ and $7.7 \% \mathrm{GU}$ ) which take longer to resolve; in our study up to 74 months from treatment. Further reporting of toxicity rates with more than 5 years of follow up are warranted.

A unique feature of the current trial is its inclusion of pelvic nodal treatment utilizing a SIB technique. While this technique for treating pelvic nodes was first pioneered in the RTOG 0529 trial in 2005 in the setting of anal cancer ${ }^{17}$, our trial was one of few to include SIB of pelvic nodes in the prostate cancer setting and is one of only three trials so far with median follow up of 5 years or longer. ${ }^{18-20}$ Although the 
benefit of pelvic nodal treatment has yet to be established, it may play a larger role in the management of prostate cancer as recent RTOG clinical trials continue to accrue and completed trials' data mature. ${ }^{21,22}$ It would be expected to see higher rates of late GI toxicity in the nodal treatment cohort, however we instead saw a trend towards less toxicity. In addition, we saw a trend towards lower rates of late GU toxicity in the nodal treatment arm. This is largely a spurious finding in the current trial, driven by small sample size and a select number of patients in the non-nodal treatment group which consistently demonstrated GI and GU toxicity over multiple time points. At the least, this toxicity data indicates that SIB treatment of pelvic lymph nodes in prostate cancer appears safe.

The largest phase III trial to include pelvic nodal treatment in testing moderate hypofractionation was the Fox Chase Cancer Center (FCCC) study, which treated $31.7 \%$ of its patients with pelvic nodal fields. 9,19 151 patients were enrolled on the hypofractionation arm with a higher proportion of high risk patients and thus ADT use than the MDACC trial (14.6\% LR, 57\% IR, 28.5\% HR, 45\% ADT), but still less use than the current trial (80\%). The pelvis was treated to $50-52 \mathrm{~Gy}$ in 26 fractions at $1.92 \mathrm{~Gy}$ per fraction and $70.2 \mathrm{~Gy}$ in 26 fractions of $2.7 \mathrm{~Gy}$ to the prostate. PTV expansions in the hypofractionation arm was $7 \mathrm{~mm}$ in all directions except for $3 \mathrm{~mm}$ posteriorly, the same as the current protocol. Updated results show no significant difference in any QOL measures using EPIC, IPSS, and EQD5 scores, but interestingly a trend towards a worse rate of distant metastases at 10 years in the hypofractionation arm. ${ }^{9,23}$

Di Muzio et al. also reported 5 year results of a large phase $\mathrm{l} / \mathrm{Il}$ trial involving pelvic lymph node coverage in intermediate and high risk patients. ${ }^{18}$ Fifty-three intermediate risk and 80 high risk patients received $74.2 \mathrm{~Gy}$ in 28 fractions of $2.65 \mathrm{~Gy}$ and $51.8 \mathrm{~Gy}$ at $1.85 \mathrm{~Gy}$ per fraction to the pelvic lymph nodes. PTV expansion was 10-mm in all directions except $8 \mathrm{~mm}$ posteriorly. The entire seminal vesicles were covered in all patients, with a risk adapted approach to dose. ADT was used in $70 \%$ of patients. Cumulative rates of grade 2 and $3 \mathrm{GU}$ toxicities were $20.2 \%$ and $5.9 \%$ respectively, with cumulative rates of grade 2 and 3 GI toxicities $17 \%$ and $6.3 \%$ respectively. While our rates of GU toxicities are comparable, our rate of GI toxicity is numerically lower and could be explained by larger PTV expansions or complete seminal vesicle coverage in the Di Muzio trial.

There have been other smaller trials confirming that SIB treatment of pelvic lymph nodes is safe, at various fraction sizes from $1.56 \mathrm{~Gy}$ to $2 \mathrm{~Gy}$ (total doses from $50 \mathrm{~Gy}$ to $56 \mathrm{~Gy}$ ) with all trials showing acute grade 3 or higher $\mathrm{GI}$ toxicity $<3 \%$ and late grade 3 or higher $\mathrm{GI}$ toxicity $<10 \% .{ }^{20,24-30}$

We must exercise caution when attempting to identify the optimal regimen of moderate hypofractionation. Given the considerable heterogeneity in dose-fractionation it is difficult to compare toxicity across trials. There was heterogeneity among the control arms of these trials, with some using non dose-escalated radiotherapy ${ }^{31,32}$ and others using dose-escalated radiotherapy. ${ }^{4-12,23,33}$ Trials were also designed differently, varying between dose-fractionation schemes to be isoeffective to late tissue effects versus dose-escalated or non dose-escalated, complicating late toxicity analysis. Trials differed in PTV margins, extent of seminal vesicle coverage in CTV, and ADT use, all of which in a recent metaanalysis have been shown to be significantly correlated with worse acute and late GI toxicity. ${ }^{3}$ In addition, 
there were differences in quality of life instruments, definitions for assigning grade $2 \mathrm{GI}$ or GU toxicity, image guidance, and dose constraints.

Biologically effective doses (BEDs) also varied widely between hypofractionation trials, from 156 to $211 \mathrm{~Gy}$ assuming an alpha/beta ratio of 1.5, and between 85 to $108 \mathrm{~Gy}$ assuming an alpha/beta ratio of 5 , which are values typical for prostate tumor control and for late rectal toxicity respectively. ${ }^{34-40}$ Brenner and Hall discuss that the trials with the highest $\mathrm{BED}_{1.5}$ also saw significant late $\mathrm{GI}$ and $\mathrm{GU}$ toxicity, and that $\mathrm{BED}_{1.5}$ for moderately hypofractionated radiotherapy should not exceed $183 \mathrm{~Gy} .{ }^{40}$ The HYPRO trial had the highest $\mathrm{BED}_{1.5}(211 \mathrm{~Gy})$ prescribed and saw increased cumulative rates of late grade 3 or higher GU toxicity higher in the hypofractionation arm (19.0\% vs $12.9 \%)$, with toxicity non-inferiority unable to be confirmed. ${ }^{41}$ RTOG 0415 (BED $\left.1.5186 \mathrm{~Gy}\right)$, the second highest value, also showed higher late grade 2 or 3 GI and GU toxicity (HR 1.31-1.59). ${ }^{10}$ The CHHiP and PROFIT trials used the lowest BED 1.5 of moderate hypofraction trials $\left(\mathrm{BED}_{1.5} 180\right)$ and both saw no increased risk of late $\mathrm{Gl}$ or GU toxicity. ${ }^{4,5}$

Our trial shares commonalities amongst other moderate hypofractionation trials. The MD Anderson Cancer Center (MDACC) trial utilized the same fraction as IR and HR patients on our study, receiving $72 \mathrm{~Gy}$ to the prostate in 30 fractions of $2.4 \mathrm{~Gy}$ each at a BED of $187 \mathrm{~Gy} .{ }^{12}$ This trial had 102 patients on their hypofractionation arm utilizing IMRT technique, consisting of $27 \%$ low, $72 \%$ intermediate, and $1 \%$ high risk. Only the prostate and proximal SV was treated. The protocol mandated that PTV expansion was 10 to $15 \mathrm{~mm}$ in all dimensions except for 4 to $8 \mathrm{~mm}$ posteriorly, compared to $7 \mathrm{~mm}$ in all directions except for $3 \mathrm{~mm}$ posteriorly in the current protocol. Approximately $20 \%$ of patients received ADT, compared to $80 \%$ on our trial. Late GU (grade 2 or 3 ) toxicity was no different between arms, with $16.4 \%$ in the conventional arm and $15.1 \%$ in the hypofractionated arm at 8 years. There was a trend $(p=0.08)$ towards worse late (grade 2 or 3) GI toxicity in the moderate hypofractionation arm, up to $12.6 \%$ compared to $5 \%$ at 8 years. This however could be reduced to $8.6 \%$ if $\mathrm{V} 65<15 \%$. These values compare favorably to our grade 2 or higher GI toxicity $(5.8 \%)$ and GU toxicity $(7.7 \%)$ at 5 years.

The current clinical trial is ultimately limited by multiple factors, including its phase $\mathrm{I} / \mathrm{Il}$ design and low patient numbers. In addition, we did not utilize a patient-reported quality of life instrument for GI toxicity. Due to sample size, we were unable to make specific conclusions about toxicity differences between pelvic nodal and prostate only patients. Like most moderate hypofractionation trials, our follow up is limited to 5 years at present.

\section{Conclusions}

The results from this trial add to the growing body of maturing data with 5 or more years of follow with moderately hypofractionated courses of prostate radiotherapy. The data demonstrate similar rates of late toxicity with or without pelvic nodal irradiation. The use of $2.4 \mathrm{~Gy} /$ day to the prostate also produces similar biochemical control and acceptable acute/late toxicity when compared to standard fractionation, justifying its appropriateness for routine use in clinical practice. 


\section{Abbreviations}

- $\mathrm{IMRT}=$ intensity modulated radiation therapy

- $\mathrm{SIB}=$ simultaneous integrated boost

- $\mathrm{LN}=$ lymph node

- $\mathrm{LR}=$ low risk

- $\mathrm{IR}=$ intermediate risk

- $\mathrm{HR}=$ high risk

- EBRT = external beam radiation therapy

- $\mathrm{GI}=$ gastrointestinal

- $\mathrm{GU}=$ genitourinary

- $\mathrm{PCSM}=$ prostate cancer specific mortality

- ASTRO = American Society for Radiation Oncology

- $\mathrm{ASCO}=$ American Society of Clinical Oncology

- $\mathrm{AUA}=$ American Urological Association

- $\mathrm{SV}=$ seminal vesicle

- KPS = Karnofsky performance status

- PSA = prostate specific antigen

- $\mathrm{NCCN}=$ National Comprehensive Cancer Network

- $\mathrm{ADT}=$ androgen deprivation therapy

- $\mathrm{CTV}=$ clinical target volume

- $\mathrm{PTV}=$ planning target volume

- $\mathrm{OAR}=$ organ at risk

- $\mathrm{CT}$ = computed tomography

- bRFS = biochemical relapse free survival

- $\mathrm{CTCAE}=$ Common Terminology Criteria for Adverse Events

- IPSS = International Prostate Symptoms Score

- IIEF = international index of erectile function

- $\mathrm{BED}=$ biologically effective dose

\section{Clinical Trial Registration Information}

- Registry: ClinicalTrials.gov

- Trial Registration Number: NCT01117935

- Date of Registration: 5/6/2010 
- URL of trial registry record: https://clinicaltrials.gov/ct2/show/NCT01117935

\section{Declarations}

- Ethics approval and consent to participate: This study was reviewed in the Massey Cancer Center and Hunter-Holmes McGuire Veterans Administration Medical Center Institutional Review Boards and approved.

- Consent for publication: Not applicable

- Availability of data and material: The datasets during and/or analyzed during the current study available from the corresponding author on reasonable request.

- Competing interests: None.

- Funding: This study was supported, in part, with funding from the National Institutes of HealthNational Cancer Institute Cancer Center Support Grant P30 CA016059, the Massey Cancer Center, and the Hunter-Holmes McGuire Veterans Administration Medical Center.

- Authors' contributions: MC, MA, and DM conceived of the project and worked towards securing IRB approval and funding. MC, DH, and VS performed data-collection. NM and XD performed statistical analysis. AR wrote the initial manuscript drafts. SS, DM, MA, and MC provided editorial input.

- Acknowledgements: Not applicable

\section{References}

1. Pasalic D, Kuban DA, Allen PK, Tang C. Dose Escalation for Prostate Adenocarcinoma: A Long-Term Update on the Outcomes of a Phase 3, Single Institution Randomized Clinical Trial. International Journal of (2019).

2. Michalski JM, et al. Effect of Standard vs Dose-Escalated Radiation Therapy for Patients With Intermediate-Risk Prostate Cancer: The NRG Oncology RTOG 0126 Randomized Clinical Trial. JAMA Oncol. 2018. doi:10.1001/jamaoncol.2018.0039.

3. Datta NR, Stutz E, Rogers S, Bodis S. Conventional Versus Hypofractionated Radiation Therapy for Localized or Locally Advanced Prostate Cancer: A Systematic Review and Meta-analysis along with Therapeutic Implications. Int J Radiat Oncol Biol Phys. 2017;99:573-89.

4. Catton CN, et al. Randomized Trial of a Hypofractionated Radiation Regimen for the Treatment of Localized Prostate Cancer. J Clin Oncol. 2017;35:1884-90.

5. Lancet (2016).

6. Staffurth J, et al. Impact of Prostate Cancer Hypofractionation on Patient Reported Outcomes:

Baseline to 5 Years Change in the CHHIP Trial. Int J Radiat Oncol Biol Phys. 2018;102:1-2.

7. Arcangeli G, et al. Moderate Hypofractionation in High-Risk, Organ-Confined Prostate Cancer: Final Results of a Phase III Randomized Trial. J Clin Oncol. 2017;35:1891-7. 
8. Wortel RC, Oomen-de Hoop E, Heemsbergen WD, Pos FJ, Incrocci L. Moderate Hypofractionation in Intermediate- and High-Risk, Localized Prostate Cancer: Health-Related Quality of Life From the Randomized, Phase 3 HYPRO Trial. Int J Radiat Oncol Biol Phys. 2019;103:823-33.

9. Avkshtol V, et al. 10-Year Update of a Randomized Prospective Trial of Conventional Versus Hypofractionated Radiation Therapy for Localized Prostate Cancer. Int J Radiat Oncol Biol Phys. 2018;102:30-1.

10. Lee WR, et al. NRG Oncology RTOG 0415: A randomized phase III non-inferiority study comparing two fractionation schedules in patients with low-risk prostate cancer. J Clin Oncol. 2016;34:1.

11. Bruner DW, et al. Quality of Life in Patients With Low-Risk Prostate Cancer Treated With Hypofractionated vs Conventional Radiotherapy: A Phase 3 Randomized Clinical Trial. JAMA Oncol. 2019. doi:10.1001/jamaoncol.2018.6752.

12. Hoffman KE, et al. Randomized Trial of Hypofractionated, Dose-Escalated, Intensity-Modulated Radiation Therapy (IMRT) Versus Conventionally Fractionated IMRT for Localized Prostate Cancer. J Clin Oncol. 2018;36:2943-9.

13. Niazi TM, et al. Phase 3 Study of Hypofractionated, Dose Escalation Radiation Therapy for High-Risk Adenocarcinoma of the Prostate. Int J Radiat Oncol Biol Phys. 2017;99:130-1.

14. Morgan SC, et al. Hypofractionated Radiation Therapy for Localized Prostate Cancer: An ASTRO, ASCO, and AUA Evidence-Based Guideline. J. Clin. Oncol. JC01801097 (2018).

15. Roach $\mathrm{M}$, et al. Predicting the risk of lymph node involvement using the pre-treatment prostate specific antigen and Gleason score in men with clinically localized prostate cancer. Int J Radiat Oncol Biol Phys. 1994;28:33-7.

16. Gay HA, et al. Pelvic normal tissue contouring guidelines for radiation therapy: a Radiation Therapy Oncology Group consensus panel atlas. Int J Radiat Oncol Biol Phys. 2012;83:e353-62.

17. Kachnic LA, et al. RTOG 0529: a phase 2 evaluation of dose-painted intensity modulated radiation therapy in combination with 5-fluorouracil and mitomycin-C for the reduction of acute morbidity in carcinoma of the anal canal. Int J Radiat Oncol Biol Phys. 2013;86:27-33.

18. Di Muzio NG, et al. Moderate Hypofractionation with Simultaneous Integrated Boost in Prostate Cancer: Long-term Results of a Phase I-II Study. Clin Oncol. 2016;28:490-500.

19. Pollack $A$, et al. Randomized trial of hypofractionated external-beam radiotherapy for prostate cancer. J Clin Oncol. 2013;31:3860-8.

20. Magli A, et al. Hypofractionated simultaneous integrated boost (IMRT-SIB) with pelvic nodal irradiation and concurrent androgen deprivation therapy for high-risk prostate cancer: results of a prospective phase II trial. Prostate Cancer Prostatic Dis. 2018;21:269-76.

21. Pollack A, et al. Short Term Androgen Deprivation Therapy Without or With Pelvic Lymph Node Treatment Added to Prostate Bed Only Salvage Radiotherapy: The NRG Oncology/RTOG 0534 SPPORT Trial. Int J Radiat Oncol Biol Phys. 2018;102:1605.

22. Androgen-Deprivation Therapy and Radiation Therapy in Treating Patients With Prostate Cancer Full. Text View - ClinicalTrials.gov. https://clinicaltrials.gov/ct2/show/NCT01368588. 
23. Shaikh T, et al. Long-Term Patient-Reported Outcomes From a Phase 3 Randomized Prospective Trial of Conventional Versus Hypofractionated Radiation Therapy for Localized Prostate Cancer. Int J Radiat Oncol Biol Phys. 2017;97:722-31.

24. McCammon R, et al. Toxicity assessment of pelvic intensity-modulated radiotherapy with hypofractionated simultaneous integrated boost to prostate for intermediate- and high-risk prostate cancer. Int J Radiat Oncol Biol Phys. 2009;75:413-20.

25. Engels B, et al. Helical tomotherapy with simultaneous integrated boost for high-risk and lymph node-positive prostate cancer: early report on acute and late toxicity. Technol Cancer Res Treat. 2009;8:353-9.

26. Adkison JB, et al. Phase I trial of pelvic nodal dose escalation with hypofractionated IMRT for highrisk prostate cancer. Int J Radiat Oncol Biol Phys. 2012;82:184-90.

27. Alongi $F$, et al. Moderate hypofractionation and simultaneous integrated boost with volumetric modulated arc therapy (RapidArc) for prostate cancer. Strahlenther Onkol. 2012;188:990-6.

28. Saracino B, et al. Intensity-modulated pelvic radiation therapy and simultaneous integrated boost to the prostate area in patients with high-risk prostate cancer: a preliminary report of disease control. Cancer Med. 2014;3:1313-21.

29. Franzese $C$, et al. Moderate hypofractionated radiotherapy with volumetric modulated arc therapy and simultaneous integrated boost for pelvic irradiation in prostate cancer. J Cancer Res Clin Oncol. 2017;143:1301-9.

30. Jorgo K, et al. Acute and Late Toxicity after Moderate Hypofractionation with Simultaneous Integrated Boost (SIB) Radiation Therapy for Prostate Cancer. A Single Institution, Prospective Study. Pathol Oncol Res. 2019. doi:10.1007/s12253-019-00623-2.

31. Yeoh EE, et al. Hypofractionated versus conventionally fractionated radiation therapy for prostate carcinoma: updated results of a phase III randomized trial. Int J Radiat Oncol Biol Phys. 2006;66:1072-83.

32. Lukka $\mathrm{H}$, et al. Randomized trial comparing two fractionation schedules for patients with localized prostate cancer. vol. 23 6132-6138 http://dx.doi.org/10.1200/JC0.2005.06.153 (2005).

33. Incrocci $L$, et al. Hypofractionated versus conventionally fractionated radiotherapy for patients with localised prostate cancer (HYPRO): final efficacy results from a randomised, multicentre, open-label, phase 3 trial. Lancet Oncol. 2016;17:1061-9.

34. Tucker SL, et al. Estimation of $\alpha / \beta$ for late rectal toxicity based on RTOG $94-06$. Int J Radiat Oncol Biol Phys. 2011;81:600-5.

35. Brenner DJ. Fractionation and late rectal toxicity. Int J Radiat Oncol Biol Phys. 2004;60:1013-5.

36. Brenner DJ, et al. Direct evidence that prostate tumors show high sensitivity to fractionation (low $\mathrm{a} / \beta$ ratio), similar to late-responding normal tissue. International Journal of Radiation Oncology*Biology*Physics. 2002;52:6-13.

37. Proust-Lima $C$, et al. Confirmation of a low $\alpha / \beta$ ratio for prostate cancer treated by external beam radiation therapy alone using a post-treatment repeated-measures model for PSA dynamics. Int $J$ 
Radiat Oncol Biol Phys. 2011;79:195-201.

38. Miralbell R, Roberts SA, Zubizarreta E, Hendry JH. Dose-fractionation sensitivity of prostate cancer deduced from radiotherapy outcomes of 5,969 patients in seven international institutional datasets: $\alpha / \beta=1.4(0.9-2.2)$ Gy. Int J Radiat Oncol Biol Phys. 2012;82:e17-24.

39. Vogelius IR, Bentzen SM. Meta-analysis of the alpha/beta ratio for prostate cancer in the presence of an overall time factor: bad news, good news, or no news? Int J Radiat Oncol Biol Phys. 2013;85:8994.

40. Brenner DJ, Hall EJ. Are We Now Able to Define Guidelines for Moderate Hypofractionation in Prostate Cancer Radiation Therapy? Int J Radiat Oncol Biol Phys. 2018;100:871-3.

41. Aluwini S, et al. Hypofractionated versus conventionally fractionated radiotherapy for patients with prostate cancer (HYPRO): late toxicity results from a randomised, non-inferiority, phase 3 trial. Lancet Oncol. 2016;17:464-74.

\section{Tables}

Table 1. Target and normal tissue dose constraints 


\begin{tabular}{|c|c|c|c|}
\hline Target Tissue & Dose (Gy) & Goal $\geq(\%)$ & Protocol Violation \% \\
\hline Prostate PTV - Low risk & $69.6 / 29 \mathrm{Fx}$ & 95 & $<90$ \\
\hline Prostate PTV - Intermediate/high risk & $72 / 30 \mathrm{Fx}$ & 95 & $<90$ \\
\hline Seminal vesicle PTV & $54 / 30 \mathrm{Fx}$ & 95 & $<90$ \\
\hline Lymph node PTV & $50.4 / 30 \mathrm{Fx}$ & 95 & $<90$ \\
\hline Organ at Risk & Dose (Gy) & Goal $\leq(\%)$ & $\geq(\%)$ \\
\hline Rectum & 36 & 50 & 60 \\
\hline Rectum & 54 & 30 & 40 \\
\hline Rectum & 66 & 20 & 30 \\
\hline Rectum & 72 & 5 & 15 \\
\hline Rectum & 70 & $<10 \mathrm{cc}$ & $>12 \mathrm{cc}$ \\
\hline Posterior half of rectum & 45 & 2 & 12 \\
\hline Small bowel & 25 & 50 & 60 \\
\hline Small bowel & 45 & 33 & 43 \\
\hline Small bowel & 52 & 2 & 12 \\
\hline Bladder & 50 & 50 & 60 \\
\hline Bladder & 64 & 25 & 35 \\
\hline Bladder & 72 & 3 & 13 \\
\hline Non-PTV bladder & 72 & 3 & 13 \\
\hline Femoral heads & 35 & 50 & 60 \\
\hline Femoral heads & 40 & 10 & 20 \\
\hline Femoral heads & 45 & 2 & 12 \\
\hline Penile bulb* & 50 & 35 & $\mathrm{n} / \mathrm{a}$ \\
\hline Skin $1 \mathrm{~cm}$ & 30 & 50 & 60 \\
\hline Skin $1 \mathrm{~cm}$ & 35 & 10 & 20 \\
\hline Skin $1 \mathrm{~cm}$ & 45 & 2 & 12 \\
\hline
\end{tabular}

Table 2. Patient Characteristics* 


\begin{tabular}{|c|c|}
\hline Variable & Number of patients (percentage) \\
\hline Patients evaluable & 52 \\
\hline Age & $65(51-80)$ \\
\hline \multicolumn{2}{|l|}{ Race } \\
\hline African American & $28(53.8 \%)$ \\
\hline White (non-Hispanic) & $24(46.2 \%)$ \\
\hline \multicolumn{2}{|l|}{ Gleason Score } \\
\hline 6 & $14(27.5 \%)$ \\
\hline $7(3+4)$ & 17 \\
\hline $7(4+3)$ & 2 \\
\hline 8 & $9(17.7 \%)$ \\
\hline 9 & $9(17.7 \%)$ \\
\hline 10 & $1(2.0 \%)$ \\
\hline \multicolumn{2}{|l|}{ Clinical T Stage } \\
\hline $\mathrm{T} 1$ & 40 \\
\hline $\mathrm{T} 2 \mathrm{a}$ or $\mathrm{T} 2 \mathrm{~b}$ & 7 \\
\hline $\mathrm{T} 2 \mathrm{c}$ & 1 \\
\hline T3 & 3 \\
\hline $\mathrm{T} 4$ & 0 \\
\hline \multicolumn{2}{|l|}{ PSA } \\
\hline iPSA* & $6.6(2-147)$ \\
\hline $\mathrm{iPSA} \leq 10$ & $31(59.6 \%)$ \\
\hline iPSA $>10, \leq 20$ & $12(23.1 \%)$ \\
\hline iPSA $>20$ & $9(17.3 \%)$ \\
\hline \multicolumn{2}{|l|}{ Risk Classification } \\
\hline Low & $11(20 \%)$ \\
\hline Intermediate & $23(41.8 \%)$ \\
\hline High & $21(38.2 \%)$ \\
\hline \multicolumn{2}{|l|}{$\mathrm{ADT}$} \\
\hline Yes & $44(80 \%)$ \\
\hline No & $11(20 \%)$ \\
\hline
\end{tabular}

$*_{\mathrm{iPSA}}=$ initial prostate-specific antigen at initiation of treatment, *median age in years (range)

Figures 
A

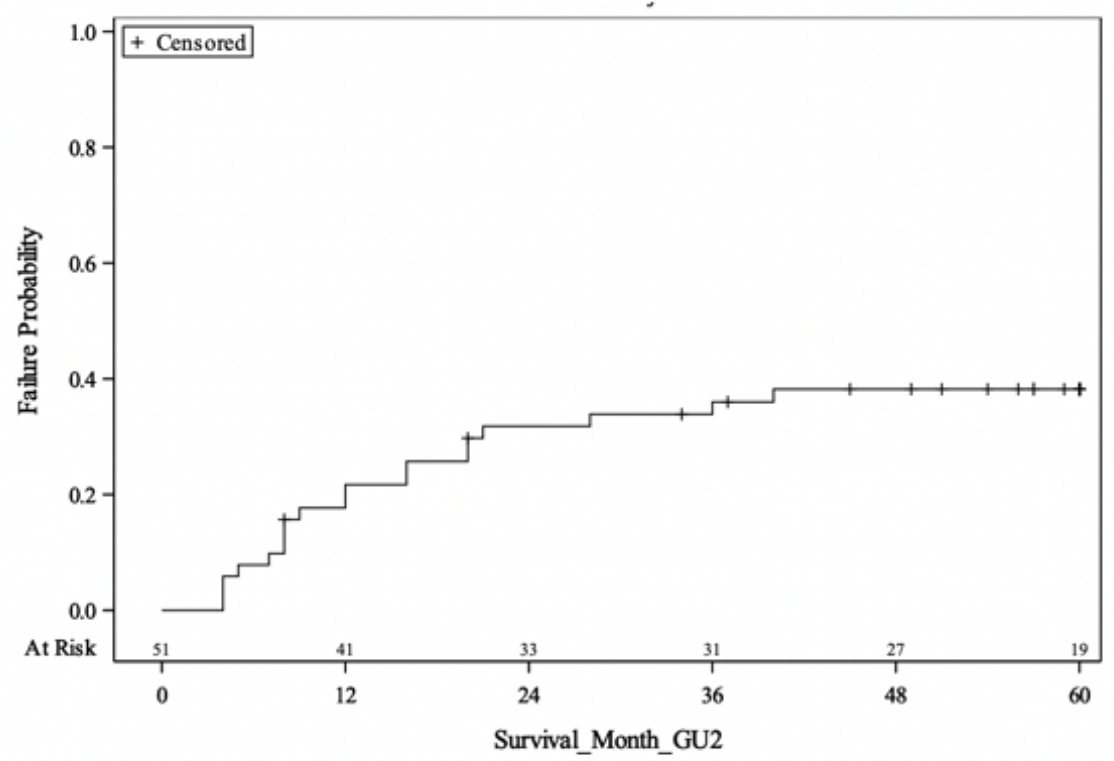

B

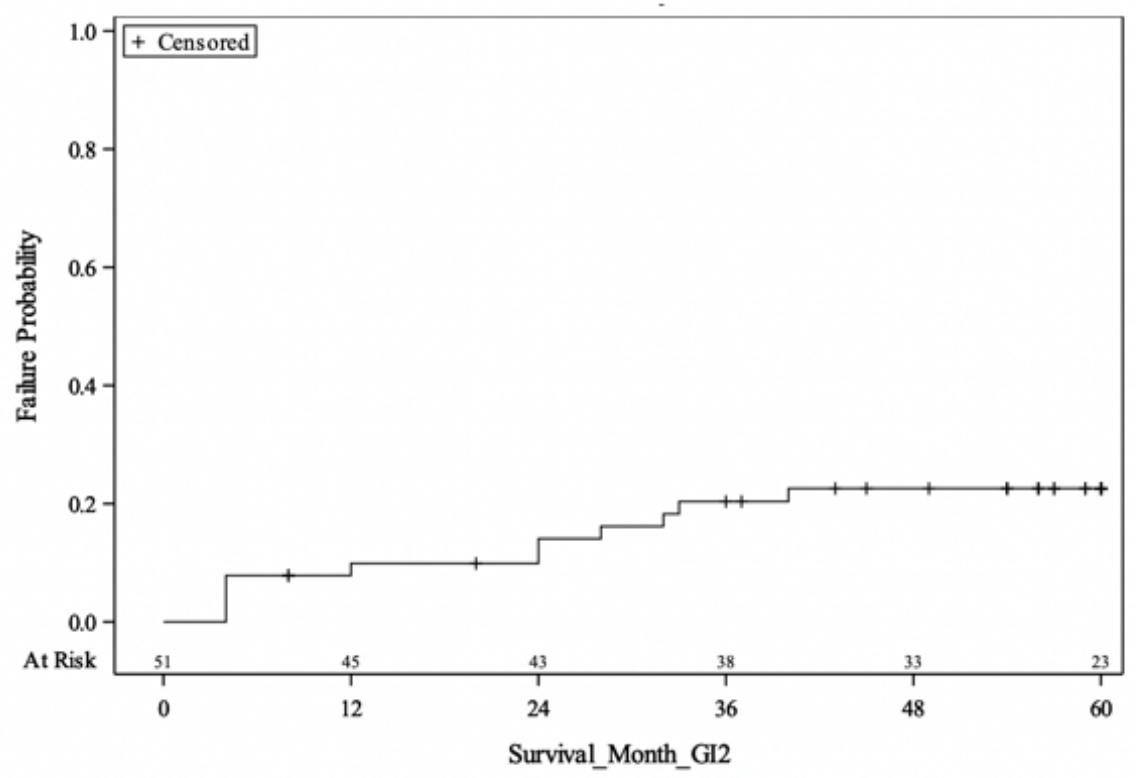

Figure 1

(A) Cumulative risk of grade 2 or higher late genitourinary (GU) toxicity (B) Cumulative risk of grade 2 or higher late gastrointestinal (GI) toxicity. 


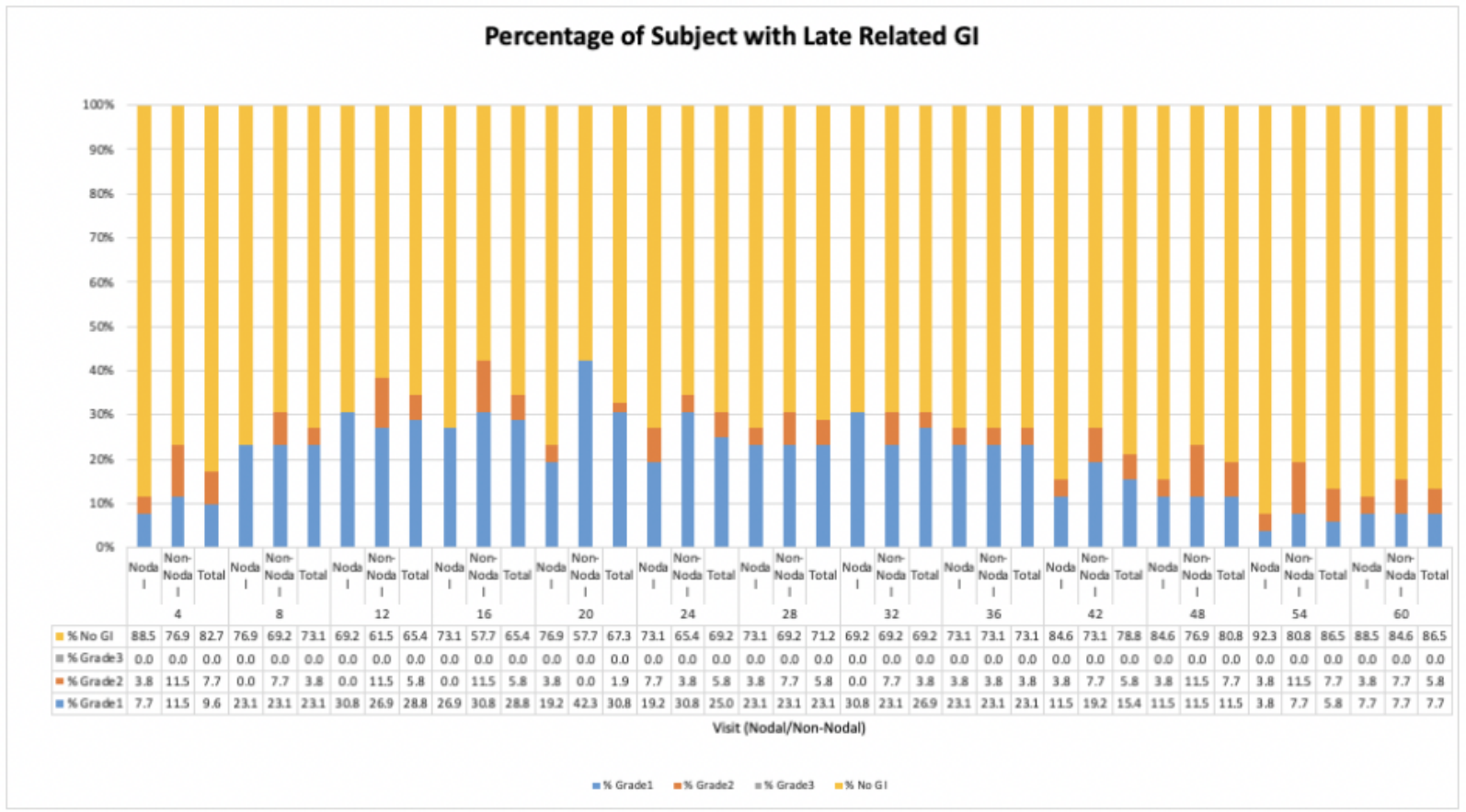

B

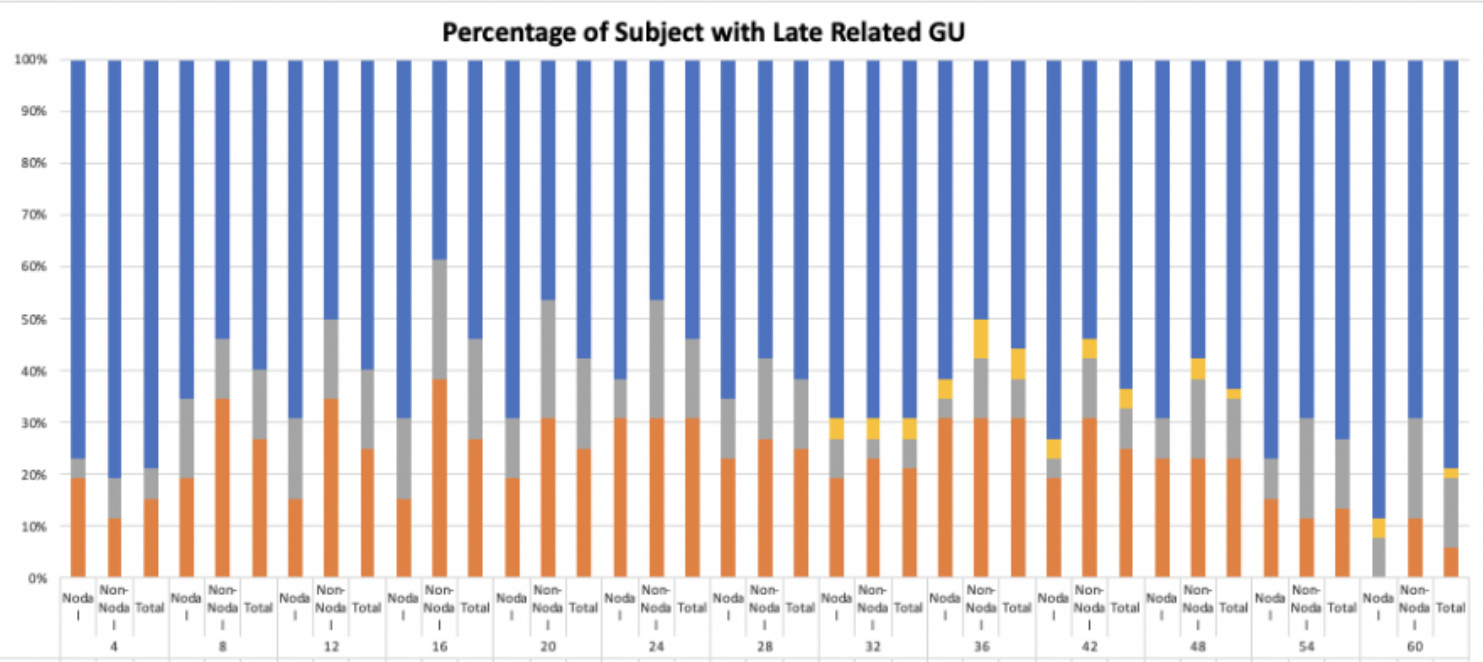

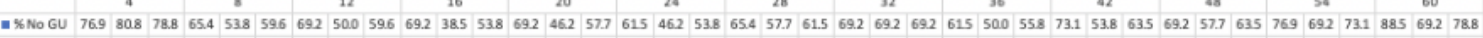

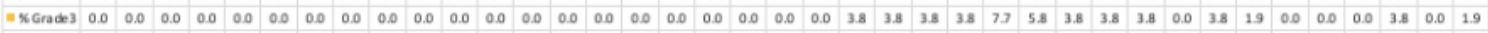

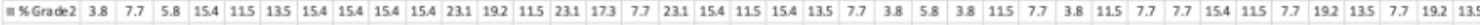

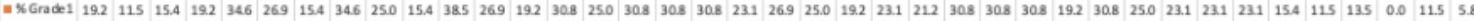
Visit (Noda/Non-Nodal)

Figure 2

(A) Percentage of Late GI related toxicity by grade (B) Percentage of Late GU related toxicity by grade 
Patient Reported AUA Score

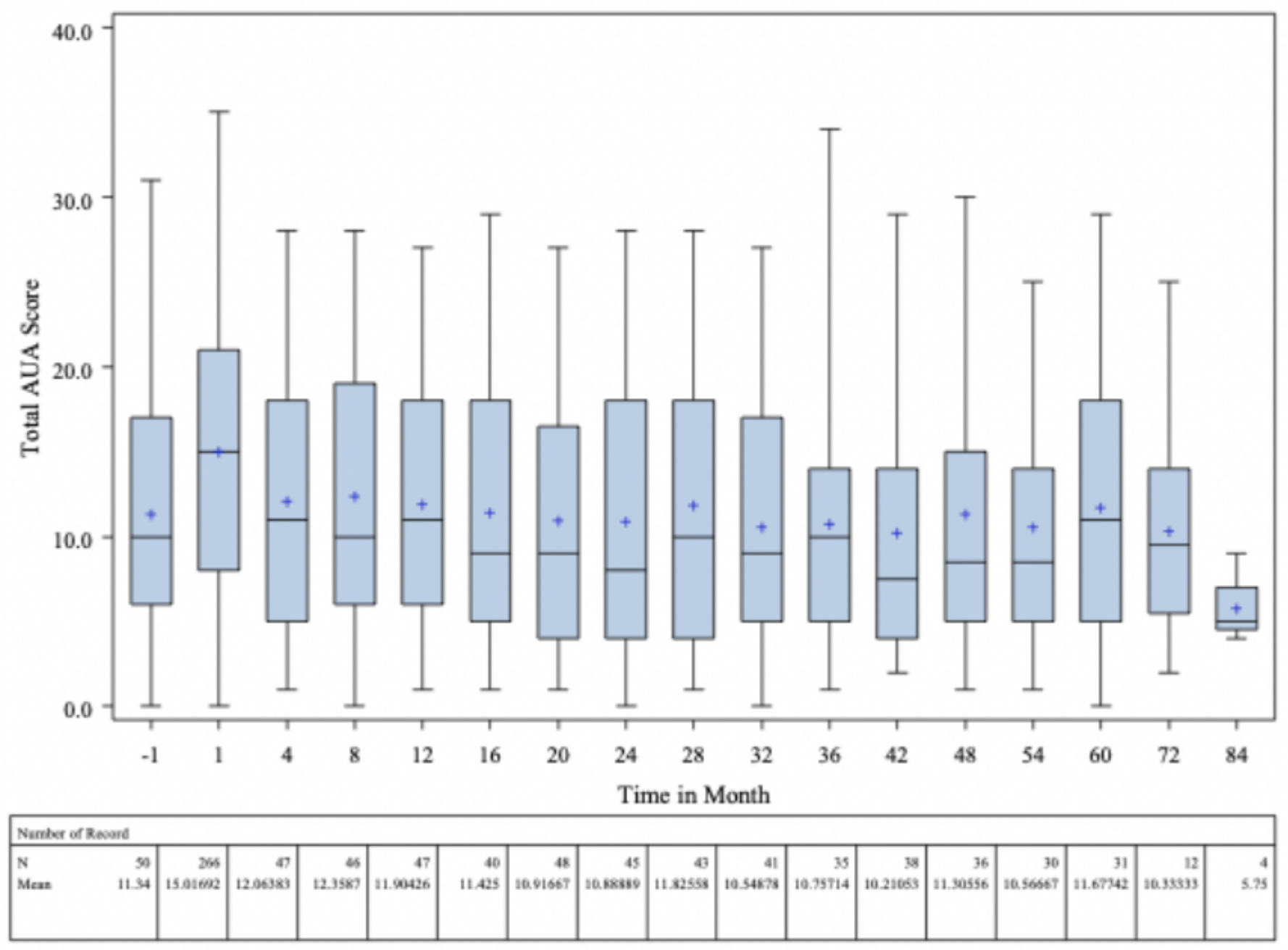

Figure 3

Patient Reported AUA Score 
Patient Reported IIEF Score

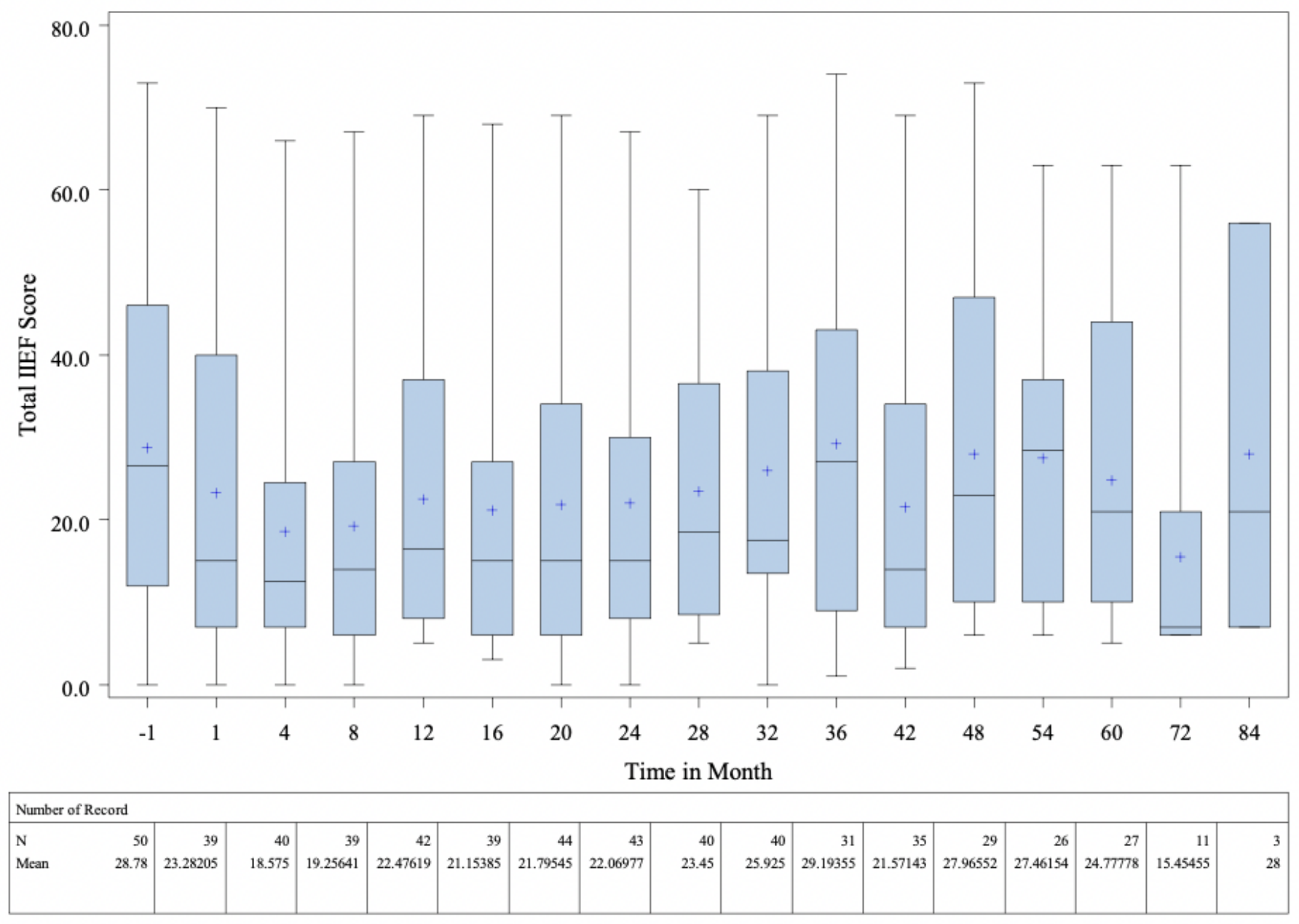

Figure 4

Patient Reported IIEF Score 


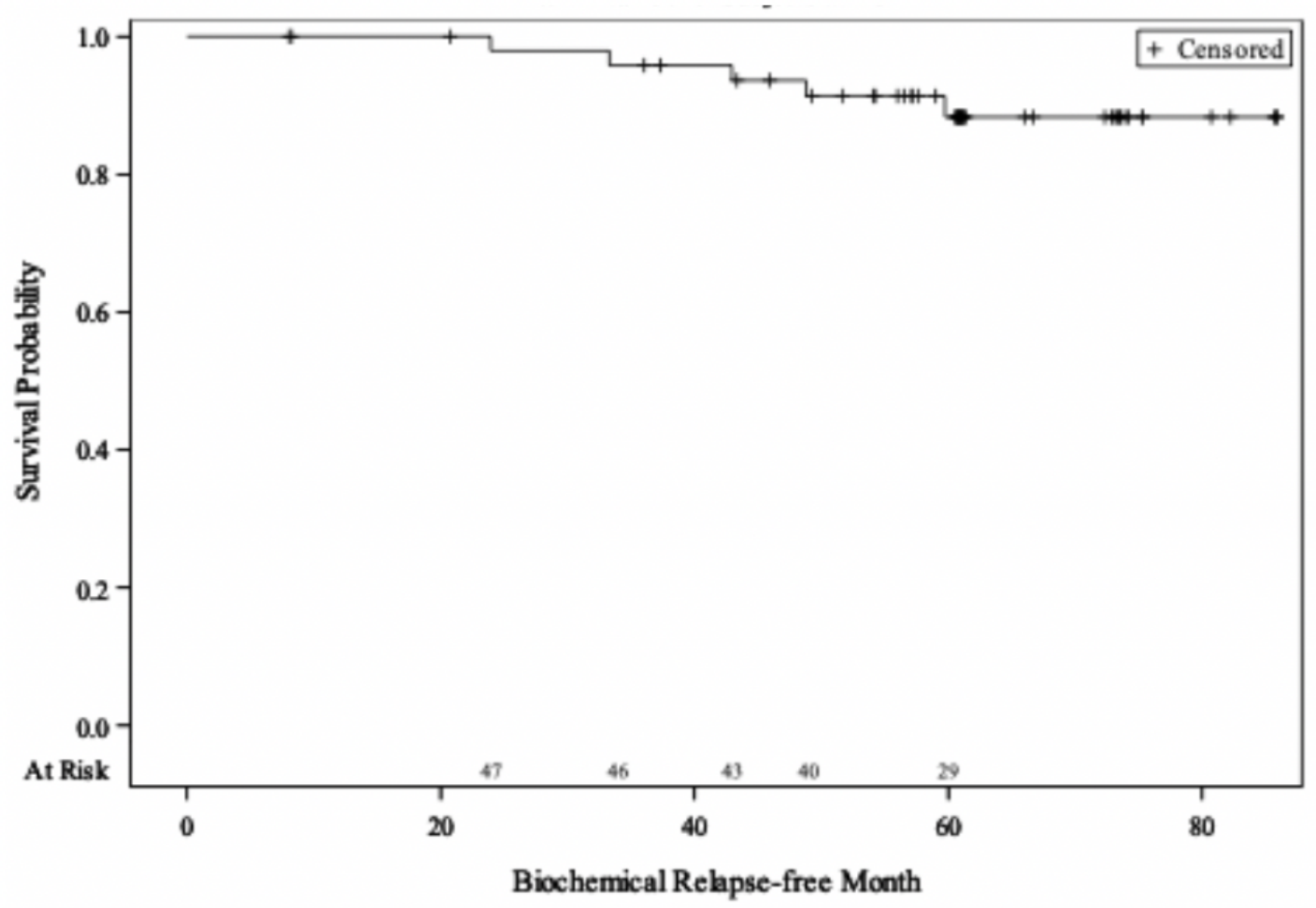

Figure 5

Biochemical Relapse Free Survival at 5 years. 